\title{
Symmetrization associated with hyperbolic reflection principle
}

\author{
Yuuki Ida* (D), Tsuyoshi Kinoshita and Tomohiro Matsumoto
}

\begin{abstract}
In this paper, in view of application to pricing of Barrier options under a stochastic volatility model, we study a reflection principle for the hyperbolic Brownian motion, and introduce a hyperbolic version of Imamura-Ishigaki-Okumura's symmetrization. Some results of numerical experiments, which imply the efficiency of the numerical scheme based on the symmetrization, are given.
\end{abstract}

Keywords: Hyperbolic Brownian motion, Reflection principle, Symmetrization, Barrier option, Euler-Maruyama scheme

\section{Introduction}

Reflection principle and the static hedge of barrier options The reflection principle of standard Brownian motion relates the probability distribution of a first hitting time to a boundary to the 1-dimensional marginal distribution of the process. The formula has a direct application in continuous-time finance, that is, the static hedging of barrier options ${ }^{1}$. The idea is explained roughly as follows. Suppose that we sold a knock-out call option ${ }^{2}$ (which is a typical barrier option). Its pay-off is described as

$$
\left(S_{T}-K\right)_{+} 1_{\{\tau>T\}},
$$

where

- $T$ is the expiry date of the option,

- $K$ is the exercise price,

- $S$ is the price process of a risky asset, with $S_{0}>K$,

and

- $\tau:=\inf \left\{s>0: S_{s}<K^{\prime}\right\}$, the first hitting time of $S$ to $K^{\prime}$, the knock-out boundary, with $K^{\prime}<K$.

The static hedge of the knock-out option consists of two plain-vanilla (=without knock-out condition) options, long position of call option with pay-off $\left(S_{T}-K\right)_{+}$, and short position of "put option" whose value

*Correspondence: ra0001pv@gmail.com

Department of Mathematics, Ritsumeikan University, 1-1-1Nojihigashi,

Kusatsu, 525-8577 Shiga, Japan
- at $\tau$ equals the call, and

- is zero at $T$ on $\tau>T$.

This simple portfolio hedges the knock-out option since it is

- zero at $T$ on $\tau \leq T$ since at $\tau$ it is liquidated, and

- $\left(S_{T}-K\right)$ at $T$ on $\tau>T$.

This relation can be expressed as

$$
\begin{aligned}
& E\left[\left(S_{T}-K\right)_{+} 1_{\{\tau>T\}} \mid \mathcal{F}_{t \wedge \tau}\right] \\
& =E\left[\left(S_{T}-K\right)_{+} \mid \mathcal{F}_{t \wedge \tau}\right] \\
& \quad-E\left[\text { "put option" } \mid \mathcal{F}_{t \wedge \tau}\right],
\end{aligned}
$$

for $0 \leq t \leq T$, where $\left\{\mathcal{F}_{t}\right\}$ is the filtration generated by $S$. The existence of such an option is ensured by the reflection principle. If $S$ is geometric Brownian motion, it can be the option with pay-off $\left(K-S_{T}\right)_{+}$since

$$
\left(S_{t}-K\right)_{\tau \leq t \leq T} \stackrel{\text { (law) }}{=}\left(K-S_{t}\right)_{\tau \leq t \leq T}
$$

by the reflection principle. In general, the property is referred to as (arithmetic) put-call symmetry at $K$ [4], which is weaker than the reflection principle that ensures put-call symmetry for any $K$.

The interpretation is first proposed in [3], and there are vast literatures since then (see e.g. [1] and references therein). Among these, we just mention a multidimensional extension proposed in [11], where the reflection principle with respect to reflection groups is applied to the pricing of multi-asset barrier options, barrier being 
the boundary of a Weyl chamber. To the best of our knowledge, it is the first attempt to deal with the cases where the barrier(=knock-out/in boundary) is not a one point set.

\section{Symmetrization and its application to numerical calculation of the price of barrier options}

A new point of view in the literature, the symmetrization, was first introduced in [10], and further generalized in [2]. The symmetrization is a procedure to convert a given diffusion into the one with a weaker version of reflection principle, aiming at obtaining a precise numerical value of the price of barrier options in a reasonable computational time, rather than static-hedge in the market described in the previous section.

Let us briefly explain their idea. We work on 1-dimensional case for simplicity. Let $S$ be a diffusion process satisfying the following stochastic differential equation:

$$
d S_{t}=\sigma\left(S_{t}\right) d W_{t}+\mu\left(S_{t}\right) d t,
$$

where, $\sigma$ and $\mu$ are piece-wise continuous functions with linear growth. In general we cannot expect the formula (1) to hold. The symmetrization $\tilde{S}$ of $S$ alternatively satisfies (1). It is defined as a (weak) solution to

$$
d \tilde{S}_{t}=\tilde{\sigma}\left(\tilde{S}_{t}\right) d W_{t}+\tilde{\mu}\left(\tilde{S}_{t}\right) d t,
$$

where

$$
\tilde{\sigma}(x)=\left\{\begin{array}{ll}
\sigma(x) & x \geq K^{\prime} \\
\sigma(2 K-x) & x<K^{\prime}
\end{array},\right.
$$

and

$$
\tilde{\mu}(x)= \begin{cases}\mu(x) & x \geq K^{\prime} \\ -\mu(2 K-x) & x<K^{\prime}\end{cases}
$$

The following is proven in [10].

Theorem 1 (Imamura-Ishigaki-Okumura [10]) The law-unique solution $\tilde{S}$ of (4) satisfies the put-call symmetry at $K$, and $\left(\tilde{S}_{t}\right)_{0 \leq t \leq \tau}$ has the same law as $\left(S_{t}\right)_{0 \leq t \leq \tau}$.

It then implies

$$
\begin{aligned}
& \mathbf{E}\left[\left(S_{T}-K\right)_{+} 1_{\{\tau>T\}}\right] \\
& =\mathbf{E}\left[\left(\tilde{S}_{T}-K\right)_{+}\right]-\mathbf{E}\left[\left(K-\tilde{S}_{T}\right)_{+}\right] .
\end{aligned}
$$

The formula (6), however, is not anymore interpreted as static hedge relation, but it has another application. The Eq. (6) now reads that

\section{- An expectation with stopping time is converted to the one without it.}

A numerical calculation of an expectation with stopping time often is a tough challenge due to its path-dependent nature. On the other hand, an expectation with respect to one dimensional marginal of a diffusion process is in most cases numerically tractable. Thus the Eq. (6) gives a new insight to the numerical analysis of barrier options/stopping times.

\section{Euler-Maruyama approximation of the price of barrier options}

The most common technique to numerically approximate an expectation with respect to a diffusion process would be so-called "Euler-Maruyama" scheme. Here we briefly recall the scheme.

An Euler-Maruyama discretization of (3) with respect to a time partition $0=t_{0}<t_{1}<\cdots<t_{n}=T$ is given by

$$
\begin{aligned}
S_{t_{0}}^{n}= & S_{0} \\
S_{t_{k+1}}^{n}= & S_{t_{k}}^{n} \\
& +\sigma\left(S_{t_{k}}\right) \Delta W_{t_{k}} \\
& \quad+\mu\left(S_{t_{k}}\right)\left(t_{k+1}-t_{k}\right), \\
k= & 0,1, \cdots, n-1,
\end{aligned}
$$

where $\Delta W_{t_{k}}^{n} \sim N\left(0, t_{k+1}-t_{k}\right)$, mutually independent for $k=0,1, \cdots, n-1$. The stopping time $\tau$ is also approximated by

$$
\tau^{n}:=\min \left\{j: S_{t_{j+1}}^{n}<K^{\prime}\right\} .
$$

The expectation in the left-hand-side of (6) is approximated by (Monte-Carlo simulation of)

$$
\mathbf{E}\left[\left(S_{T}^{n}-K\right)_{+} 1_{\left\{\tau^{n}>T\right\}}\right],
$$

while the right-hand-side counterpart is

$$
\mathbf{E}\left[\left(\tilde{S}_{T}^{n}-K\right)_{+}\right]-\mathbf{E}\left[\left(K-\tilde{S}_{T}^{n}\right)_{+}\right],
$$

where $\tilde{S}^{n}$ is obtained by the same procedure as (7).

The discretization error, by which we mean the difference between the true value of the expectation and its Euler-Maruyama approximation like (8) or (9), is known to be of $O\left(n^{-1 / 2}\right)$ in general when $t_{k}-t_{k-1}=T / n$ for all $k$. It is reported in [5] that the one with stopping time like (8) cannot be improved, while the one with onedimensional marginal like (9) is, provided some continuity of the coefficients, known to be of $O\left(n^{-1}\right)$.

The symmetrized drift coefficient (5) may not be continuous in general even if the original one is very smooth, and as far as we know, no existing result ensures the order is of $O\left(n^{-1}\right)$ though recently there have been several papers $([12,13]$, and [14]) to deal with discontinuous coefficients in line with the problem posed here. In [10], however, they conjecture that it is the case by performing numerical experiments.

References for more detailed and precise results of the order can be found in [10]. 


\section{SABR model and hyperbolic Brownian motion}

In the present paper, we study a hyperbolic version of the symmetrization, with a view to the application of the pricing of barrier options under SABR model, which is known to be transformed to hyperbolic Brownian motion with drift.

The SABR (stochastic alha-beta-rho) model was introduced in [6]. It is given by

$$
\begin{aligned}
& d S_{t}=v_{t} \sigma\left(S_{t}\right) d W_{t}^{1} \\
& d v_{t}=v v_{t}\left(\sqrt{1-\rho^{2}} d W_{t}^{2}+\rho d W_{t}^{1}\right),
\end{aligned}
$$

where $\left(W^{1}, W^{2}\right)$ is a two dimensional Brownian motion, $\rho \in(-1,1)$ and $v$ is a constant. We note that

- A driftless local volatility model is obtained by setting $v=0$, and

- $Z_{t}:=\psi\left(S_{t / v^{2}}, V_{t / v^{2}}\right)+\sqrt{-1} V_{t / v^{2}}$ with $\psi(x, y)=(x-\rho y) / \sqrt{1-\rho^{2}}$ is a hyperbolic Brownian motion with drift, a solution to (13) in "Hyperbolic symmetrization" section (for details see [7]).

The following is a "motto" widely accepted among researchers and practitioners in finance (see e.g. [8]): as tractability of one dimensional diffusion processes is attributed to the reduction to the standard Brownian motion with drift by the Lamperti transform, so the analysis of SABR model will be converted to that of hyperbolic Brownian motion with drift, where we can still work on symmetries from linear fractional transformations. We shall observe a realization of this idea in the present paper.

\section{The contents of the present paper}

We start with introducing a hyperbolic version of the reflection principle that parallels the one with the standard Brownian motion in "Hyperbolic reflection principle" section. We introduce in "Hyperbolic symmetrization" section a weak version of the reflection principle, which also parallels with the classical put-call symmetry. Associated symmetrization is then introduced. "Numerical experiments" section is devoted to numerical studies. As in the case of the Imamura-Ishigaki-Okumura's scheme using classical symmetrization, the error is not proven to be $O\left(n^{-1}\right)$ mathematically but the numerical results support the conjecture of the hyperbolic case as well.

\section{Hyperbolic reflection principle}

\section{Invariant property of hyperbolic Brownian motion}

A Hyperbolic Brownian motion is the unique solution to

$$
\left\{\begin{array}{l}
d X_{t}=Y_{t} d W_{t}^{1} \\
d Y_{t}=Y_{t} d W_{t}^{2}
\end{array}\right.
$$

where $W^{1}$ and $W^{2}$ are independent Brownian motions. It is defined on the upper-half plane $\mathbb{H}=\left\{(x, y) \in \mathbb{R}^{2}\right.$ : $y>0\}$ and we may and sometimes will embed it to $\mathbb{C}$ by $Z_{t}=X_{t}+i Y_{t}$, where $i=\sqrt{-1}$.

Proposition 1 Let $f: \mathbb{H} \rightarrow \mathbb{H}$ be such that $f(z):=\frac{a z+b}{c z+d}$, where $\left(\begin{array}{ll}a & b \\ c & d\end{array}\right) \in S L(2, \mathbb{R})$. Then $\left(f\left(Z_{t}\right)\right)_{t \geq 0}$ and $\left(Z_{t}\right)_{t \geq 0}$ are equivalent in law provided that $f\left(Z_{0}\right)=Z_{0}$.

Proof Since $Z_{t}=X_{t}+i Y_{t}$, using Ito's formula for $Z_{t}$,

$$
\begin{aligned}
d Z_{t} & =d X_{t}+i d Y_{t}=Y_{t}\left(d W_{t}^{1}+i d W_{t}^{2}\right) \\
& =\operatorname{Im}\left(Z_{t}\right) d W_{t}^{\mathbb{C}},
\end{aligned}
$$

where $d W_{t}^{\mathbb{C}}=d W_{t}^{1}+i d W_{t}^{2}$, which we define to be a complex Brownian motion. On the other hand, since $Z_{t}$ is a conformal martingale and $f$ is a holomorphic function, we can use Ito's formula for conformal martingales to get

$$
\begin{aligned}
d f\left(Z_{t}\right) & =\partial_{z} f\left(Z_{t}\right) d Z_{t} \\
& =\frac{1}{\left(c Z_{t}+d\right)^{2}} \operatorname{Im}\left(Z_{t}\right) d W_{t}^{\mathbb{C}} \\
& =\frac{\left|c Z_{t}+d\right|^{2}}{\left(c Z_{t}+d\right)^{2}} \operatorname{Im}\left(f\left(Z_{t}\right)\right) d W_{t}^{\mathbb{C}} \\
& =\operatorname{Im}\left(f\left(Z_{t}\right)\right) d \widetilde{W}_{t}^{\mathbb{C}},
\end{aligned}
$$

where $d \widetilde{W}_{t}^{\mathbb{C}}=\frac{\left|c Z_{t}+d\right|^{2}}{\left(c Z_{t}+d\right)^{2}} d W_{t}^{\mathbb{C}}$, which is another complex Brownian motion. Hence $Z_{t}$ and $f\left(Z_{t}\right)$ are equivalent in law if they start from the same point, as they are defined by the same SDE.

\section{Hyperbolic reflections}

Let $\mathscr{C}$ be the totality of such isometries $\pi$ on the upperhalf plane $\mathbb{H}$ that $\pi^{2}=\mathrm{Id}$ and that the invariant set $\operatorname{Inv}_{\pi}:=\{z \in \mathbb{H}: \pi(z)=z\}$ is a geodesic on $\mathbb{H}$.

\section{Proposition 2 We have that}

$$
\begin{aligned}
& \mathscr{C}=\left\{\Phi_{A} \circ \Phi_{0} \in \operatorname{Isom}(\mathbb{H}):\right. \\
& A=\left(\begin{array}{ll}
a & b \\
\frac{a^{2}-1}{b} & a
\end{array}\right),\left(\begin{array}{ll} 
\pm 1 & 0 \\
c & \pm 1
\end{array}\right) \\
& a, c \in \mathbb{R}, b \in \mathbb{R} \backslash\{0\}\},
\end{aligned}
$$

where $\Phi_{A}(z)=\frac{a z+b}{c z+d}$ for $A=\left(\begin{array}{ll}a & b \\ c & d\end{array}\right) \in \mathrm{SL}(2, \mathbb{R})$ and $\Phi_{0}(z):=-\bar{z}$.

Proof It is well-known that an isometry on $\mathbb{H}$ is either $\Phi_{A}$ or $\Phi_{A} \circ \Phi_{0}$ for some $A \in \operatorname{SL}(2, \mathbb{R})$. By the fundamental theorem of algebra, we know that the equation $\Phi_{A}(z)=z$ has at most two solutions of complex for $A \in \mathrm{SL}(2, \mathbb{R})$. So $\Phi_{A}(z) \notin \mathscr{C}$. 
For $\Phi_{A} \circ \Phi_{0} \in \operatorname{Isom}(\mathbb{H})$ and for $z=x+i y$,

$$
\left(\Phi_{A} \circ \Phi_{0}\right)^{2}(z)=\frac{\left(a^{2}-b c\right) z-b(a-d)}{c(a-d) z-\left(b c-d^{2}\right)} .
$$

By a simple calculation,

$$
\begin{aligned}
& \frac{\left(a^{2}-b c\right) z-b(a-d)}{c(a-d) z-\left(b c-d^{2}\right)}=z \\
\Longleftrightarrow & \left(a^{2}-b c\right) z-b(a-d) \\
= & c(a-d) z^{2}-\left(b c-d^{2}\right) z
\end{aligned}
$$

- If $a=d$, for any $b$ and $c,(10)$ is satisfied.

Since $a^{2}-b c=1$, we have $c=\frac{a^{2}-1}{b}$ if $b \neq 0$, that is;

$$
A=\left(\begin{array}{ll}
a & b \\
\frac{a^{2}-1}{b} & a
\end{array}\right) .
$$

For $b=0, c$ is an arbitrary real number and $a= \pm 1$ from $a^{2}=1$;

$$
A=\left(\begin{array}{cc} 
\pm 1 & 0 \\
c & \pm 1
\end{array}\right)
$$

- If $a \neq d$, the Eq. (10) is

$$
c z^{2}-(a+d) z+b=0 .
$$

We get $a=-d$ and $b=c=0$.

Finally, we should find that the invariant set is geodesic. A geodesics of upper half plane is a line perpendicular to the real line, or a half-circle orthogonal to the real line.

- If

$$
A=\left(\begin{array}{ll}
a & b \\
\frac{a^{2}-1}{b} & a
\end{array}\right),
$$

and if $a \neq \pm 1$,

$$
\begin{aligned}
& \frac{-a \bar{z}+b}{-\frac{a^{2}-1}{b} \bar{z}+a}=z, \\
\Longleftrightarrow & \left(a^{2}-1\right)|z|^{2}-a b(z+\bar{z})+b^{2}=0 .
\end{aligned}
$$

The last equation means that it is a half circle, with center $\left(\frac{a b}{a^{2}-1}, 0\right)$ and radius of $\left|\frac{b}{a^{2}-1}\right|$.

$$
\text { If } a= \pm 1 \text {, }
$$

$$
-\bar{z}+b=z \text {. }
$$

The equation means that the invariant set is lines perpendicular to the real line.

- If

$$
A=\left(\begin{array}{cc} 
\pm 1 & 0 \\
c & \pm 1
\end{array}\right)
$$

and if $c \neq 0$, without loss of generality, we may set $a=1$;

$$
\begin{gathered}
\frac{-\bar{z}}{-c \bar{z}+1}=z \\
\Longleftrightarrow-c|z|^{2}+(z+\bar{z})=0 .
\end{gathered}
$$

The invariant set is a circle with center $\left(\frac{1}{c}, 0\right)$ and the radius $\frac{1}{|c|}$.

If $c=0$, the invariant set is the lines perpendicular to the real line.

\section{Hyperbolic reflection principle}

Let $\pi \in \mathscr{C}$. Then, $\mathbb{H}=D_{+} \cup \operatorname{Inv}_{\pi} \cup D_{-}$, where $D_{ \pm}$are the connected components of $\mathbb{H} \backslash \operatorname{Inv}_{\pi}$.

Proposition 3 (Hyperbolic Reflection Principle) Let $Z_{0} \in D_{+}$and $\tau=\inf \left\{t \geq 0: Z_{t} \notin D_{+}\right\}=$ $\inf \left\{t \geq 0: Z_{t} \in \operatorname{Inv}_{\pi}\right\}$. If we put $\widetilde{\widetilde{Z}}_{t}=Z_{t} 1_{\{t<\tau\}}+$ $\pi\left(Z_{t}\right) 1_{\{t \geq \tau\}}$, then we have $\left(Z_{t}\right)=\left(\tilde{Z}_{t}\right)$ in law.

Proof It suffices to show that if $\pi$ is a reflection of $\mathbb{H}$, then $\left(\pi\left(Z_{t}\right)\right)_{t \geq 0}=\left(Z_{t}\right)_{t \geq 0}$ in law if $Z_{0} \in \operatorname{Inv}_{\pi}$ since $Z$ is a strong Markov process and $Z_{\tau} \in \operatorname{Inv}_{\pi}$. As we have seen that $\pi=\Phi_{A} \circ \Phi_{0}$ for some specific $A \in \operatorname{SL}(2, \mathbb{R})$, and by Proposition 1 , we only need to check that $\left(-\bar{Z}_{t}\right)$ is identically distributed as $\left(Z_{t}\right)$ as a stochastic process, but this is obvious since $\left(X_{t}\right)$ is identically distributed as $\left(-X_{t}\right)$.

\section{Hyperbolic symmetrization}

\section{Hyperbolic put-call symmetry}

Let $\pi \in \mathscr{C}$. Then, by Proposition 2 , we know that

$$
\pi=\Phi_{A} \circ \Phi_{0}
$$

for

$$
\begin{aligned}
& A=\left(\begin{array}{cc}
a & b \\
\frac{a^{2}-1}{b} & a
\end{array}\right),\left(\begin{array}{cc} 
\pm 1 & 0 \\
c & \pm 1
\end{array}\right) \\
& a, c \in \mathbb{R}, b \in \mathbb{R} \backslash\{0\} .
\end{aligned}
$$

A Hyperbolic Brownian motion with drift is a unique solution in $\mathbb{H}$ (if it exists) to

$$
\left\{\begin{array}{l}
d X_{t}=Y_{t} d W_{t}^{1}+\mu_{1}\left(X_{t}, Y_{t}\right) d t \\
d Y_{t}=Y_{t} d W_{t}^{2}+\mu_{2}\left(X_{t}, Y_{t}\right) d t
\end{array}\right.
$$

where $W^{1}$ and $W^{2}$ are independent Brownian motions and $\mu_{1}$ and $\mu_{2}$ are measurable functions. If we use complex coordinate, the SDE (12) is rewritten as 


$$
d Z_{t}=\operatorname{Im}\left(Z_{t}\right) d W_{t}^{\mathbb{C}}+\mu\left(Z_{t}\right) d t,
$$

where $W^{\mathbb{C}}:=W^{1}+i W^{2}$ and $\mu(Z)=\mu_{1}(\operatorname{Re}(Z), \operatorname{Im}(Z))+$ $i \mu_{2}(\operatorname{Re}(Z), \operatorname{Im}(Z))$.

Theorem 2 Let $\pi=\Phi_{A} \circ \Phi_{0} \in \mathscr{C}$ and we write

$$
A=\left(\begin{array}{ll}
a & b \\
c & d
\end{array}\right)
$$

to unify the two classes in the expression (11). Suppose that $\mu$ satisfies

$$
\mu(z)=\frac{\Phi_{0} \circ \mu \circ \pi(z)}{\left(c \Phi_{0} \circ \pi(z)+d\right)^{2}},
$$

and (13) has a unique weak solution. Then $\left(\pi\left(Z_{t}\right)\right)$ and $\left(Z_{t}\right)$ have the same law as a stochastic process, provided that $Z_{0} \in \operatorname{Inv}_{\pi}$

Proof Using Itô formula for $\pi\left(Z_{t}\right)$, we have

$$
\begin{aligned}
d \pi\left(Z_{t}\right)= & d\left(\Phi_{A} \circ \Phi_{0}\left(Z_{t}\right)\right) \\
= & \partial_{\bar{z}}\left(\Phi_{A} \circ \Phi_{0}\right)\left(Z_{t}\right) d \bar{Z}_{t} \\
= & -\frac{1}{\left(c \overline{Z_{t}}-d\right)^{2}} \operatorname{Im}\left(Z_{t}\right) d \bar{W}_{t}^{\mathbb{C}} \\
& -\frac{\overline{\mu\left(Z_{t}\right)}}{\left(-c \overline{Z_{t}}+d\right)^{2}} d t \\
= & -\frac{\left|c \overline{Z_{t}}-d\right|^{2}}{\left(c \overline{Z_{t}}-d\right)^{2}} \operatorname{Im}\left(\pi\left(Z_{t}\right)\right) d \bar{W}_{t}^{\mathbb{C}} \\
& +\frac{\Phi_{0} \circ \mu \circ \pi^{2}\left(Z_{t}\right)}{\left(c \Phi_{0} \circ \pi^{2}\left(Z_{t}\right)+d\right)^{2}} d t \\
= & \operatorname{Im}\left(\pi\left(Z_{t}\right)\right) d \widetilde{W}_{t}^{\mathbb{C}}+\mu\left(\pi\left(Z_{t}\right)\right) d t,
\end{aligned}
$$

where we have used assumption (14) in the last line and

$$
d \widetilde{W}_{t}^{\mathbb{C}}=-\frac{\left|c \overline{Z_{t}}-d\right|^{2}}{\left(c \overline{Z_{t}}-d\right)^{2}} d \bar{W}_{t}^{\mathbb{C}},
$$

which is another complex Brownian motion. Now Theorem follows by the law-uniqueness of the SDE (13).

\section{Symmetrization}

Here we present a hyperbolic version of the symmetrization introduced in [2] and [10].

Theorem 3 We keep the setting of "Hyperbolic reflection principle" section and Theorem 2 except for the drift function $\mu$. We let

$$
\tilde{\mu}(z)= \begin{cases}\mu(z) & z \in D_{+} \\ \frac{\Phi_{0} \circ \mu \circ \pi(z)}{\left(c \Phi_{0} \circ \pi(z)+d\right)^{2}}, & z \in \mathbb{H} \backslash D_{+} .\end{cases}
$$

Then, (i) the law unique solution of the SDE, if it exists,

$$
d Z_{t}=\operatorname{Im}\left(Z_{t}\right) d W^{\mathbb{C}}+\tilde{\mu}\left(Z_{t}\right) d t
$$

satisfies $\left(\pi\left(Z_{t}\right)\right)=\left(Z_{t}\right)$ in law, provided that $Z_{0} \in \operatorname{In} v_{\pi}$.

(ii) Let $Z_{0} \in D_{+}$and

$\tau=\inf \left\{t \geq 0: Z_{t} \notin D_{+}\right\}=\inf \left\{t \geq 0: Z_{t} \in \operatorname{Inv}_{\pi}\right\}$. If we put $\widetilde{Z}_{t}=Z_{t} 1_{\{t<\tau\}}+\pi\left(Z_{t}\right) 1_{\{t \geq \tau\}}$, then we have $\left(Z_{t}\right)=\left(\widetilde{Z}_{t}\right)$ in law.

(iii) [Conversion Formula] Suppose that $F$ is a bounded measurable function on $\mathbb{H}$ with support in $D_{+}$. Then,

$$
\begin{aligned}
& E\left[F\left(Z_{t}\right) 1_{\{\tau>t\}}\right] \\
& =E\left[F\left(Z_{t}\right)\right]-E\left[F\left(\pi\left(Z_{t}\right)\right)\right] .
\end{aligned}
$$

Proof (i) and (ii) are direct consequences of Theorem 2 and Proposition 3. (iii) can be proven in the same manner as in [10].

Example 1 Let $Z$ be the unique solution to (13), $\pi(z)=$ $\frac{1}{\bar{z}}$ and $\operatorname{Inv}_{\pi}=\{|z|=1\}$. We let $D_{+}:=\{z \in \mathbb{H}:|z|>1\}$ and

$$
\mu(z)=c \operatorname{Im}(z)
$$

where $c$ is a constant, then the symmetrization $\tilde{\mu}$ in Theorem 3 is

$$
\tilde{\mu}(z)= \begin{cases}c \operatorname{Im}(z) & z \in D_{+} \\ -c z^{2} \operatorname{Im}\left(\frac{1}{\bar{z}}\right) & \text { otherwise }\end{cases}
$$

\section{Numerical experiments}

In the hyperbolic symmetrization proposed in the present paper the symmetrized drift may not be continuous in general, as in the case of the symmetrization in [10]. This means that no rigorous mathematical result guarantees the efficiency - (high) order of convergence- in EulerMaruyama approximation. In [10], it is claimed, however, that numerical experiments show the efficiency. In this section we present some simulation results of the Example 1 with $c=1, t=1$, and $F(z)=(|z|-1)_{+} \wedge N$ with $N=10^{4}$, which suggest that in the hyperbolic case the conjecture is still likely to be true.

We work on Euler-Maruyama discretization scheme with Monte-Carlo simulation, described below.

1. Let $n$ be the number of discretization; we put $t_{k}=k / n, k=0,1, \cdots, n$.

2. Let $Z$ be the original process and $\tilde{Z}$ be the symmetrized one. We approximate $Z$ and $\tilde{Z}$ by 
$Z^{n}=\left(X^{n}, Y^{n}\right)$ and $\widetilde{Z}^{n}=\left(\tilde{X}^{n}, \tilde{Y}^{n}\right)$, defined as

$$
\begin{aligned}
& X_{t_{k}}^{n}-X_{t_{k-1}}^{n} \\
& =Y_{t_{k-1}}^{n} \Delta W_{t_{k}}^{n}+\mu\left(Y_{t_{k-1}}^{n}\right) n^{-1}, \\
Y_{t_{k}}^{n} & =Y_{t_{k-1}}^{n} \exp \left(\Delta W_{t_{k}}^{n}-(2 n)^{-1}\right), \\
k & =1,2, \cdots, n
\end{aligned}
$$

and

$$
\begin{aligned}
& \tilde{X}_{t_{k}}^{n}-\tilde{X}_{t_{k-1}}^{n}= \tilde{Y}_{t_{k-1}}^{n} \Delta W_{t_{k}}^{n}+\tilde{\mu}_{1} \\
&\left(\tilde{X}_{t_{k-1}}^{n}, \tilde{Y}_{t_{k-1}}^{n}\right) n^{-1}, \\
& \tilde{Y}_{t_{k}}^{n}-\tilde{Y}_{t_{k-1}}^{n}= \tilde{Y}_{t_{k-1}}^{n} \Delta W_{t_{k}}^{n}+\tilde{\mu}_{2} \\
&\left(\tilde{X}_{t_{k-1}}^{n}, \tilde{Y}_{t_{k-1}}^{n}\right) n^{-1}, \\
& k=1,2, \cdots, n,
\end{aligned}
$$

where $\tilde{\mu}_{1}$ and $\tilde{\mu}_{2}$ are such that $\tilde{\mu}=\tilde{\mu}_{1}+i \tilde{\mu}_{2}$. Here $\left\{\Delta W_{t_{k}}^{n}: k=1,2, \cdots, n\right\}$ simulates, by pseudo random numbers, independent copies of centered Gaussian random variables with variance $n^{-1}$.

3. The Monte-Carlo simulation of Path-Wise Euler-Maruyama approximation of $E\left[F\left(Z_{1}\right) 1_{\{\tau>1\}}\right]$ is obtained by

$$
\begin{aligned}
& \operatorname{PW}-\operatorname{EM}(n) \\
& :=\frac{1}{M} \sum_{m=1}^{M} F\left(Z_{1}^{n, m}\right) 1_{\left\{\tau^{n, m}>1\right\}},
\end{aligned}
$$

where $Z^{n, m}$ stands for the $m$-th simulation of $Z^{n}$, and

$$
\tau^{n, m}=\min \left\{t_{k}:\left|Z_{t_{k}}^{n, m}\right| \leq 1\right\}
$$

4. The Monte-Carlo simulation of $E\left[F\left(\widetilde{Z}_{1}\right)\right]-E\left[F\left(\pi\left(\widetilde{Z}_{1}\right)\right)\right]$ is given by

Symmetrization $(n)$

$$
:=\frac{1}{M} \sum_{m=1}^{M}\left(F\left(\widetilde{Z}_{1}^{n, m}\right)-F\left(\pi\left(\widetilde{Z}_{1}^{n, m}\right)\right)\right),
$$

5. The "true" value $\operatorname{Tr}(n)$ is set to be Symmetrization $(n)$ for some large $n$.

6. The errors are calculated accordingly as

$$
\begin{aligned}
& \text { PW EM Error }(n) \\
& :=\log |\operatorname{Tr}(n)-\operatorname{PW} \operatorname{EM}(n)|
\end{aligned}
$$

and

$$
\begin{aligned}
& \text { Sym } \operatorname{Error}(n) \\
& :=\log |\operatorname{Tr}(n)-\operatorname{Symmetrization}(n)| .
\end{aligned}
$$

The results are visualized as follows. The Figures 1 and 2 show the results when $\left(X_{0}, Y_{0}\right)=(0.75,0.7)$ and $\left(X_{0}, Y_{0}\right)=(1.0,1.0)$, respectively, and the "true" value is calculate for $n=1000$. The Tables 1 and 2 are the values of the dotted points in the Figures 1 and 2, respectively. The tangent of the regression line corresponds to the order of the convergence, which may suggest that it is of order 1 in the case of symmetrization.

\section{Endnotes}

${ }^{1} \mathrm{~A}$ barrier option is a financial derivative with an additional condition that is made active when the underlying price process goes beyond/below a certain level. For details, see e.g. [9].

${ }^{2}$ An option is called of "knock-out" type if the pay-off becomes zero if the underlying price process hits a certain value.

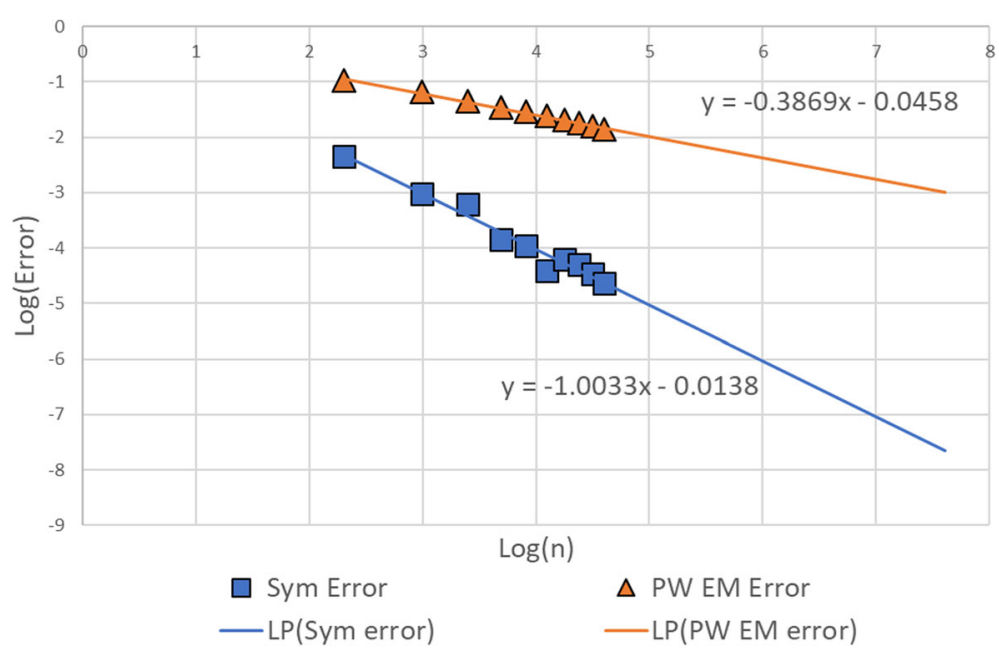

Fig. 1 The results of the first experiment 


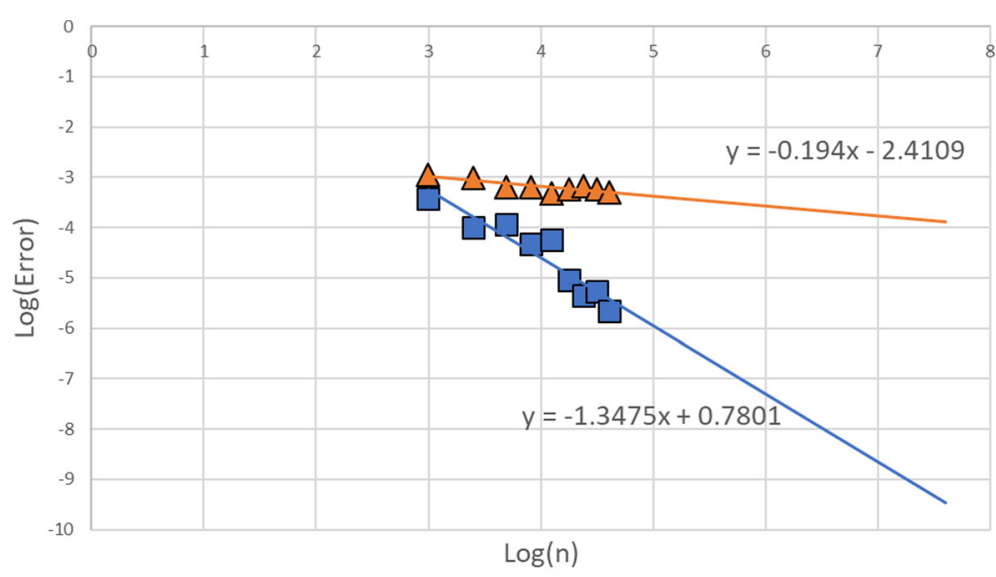

Sym Error — LP(Sym error)

$\triangle$ PW EM Error

— LP(PW EM error)

Fig. 2 The results of the second experiment

Table $1\left(X_{0}, Y_{0}\right)=(0.75,0.7), \operatorname{Tr} 1000=0.116674$

\begin{tabular}{llllll}
\hline M:No. of simmulation trials & n:No. of time steps & Symmetrization & PW EM & Sym Error & PW EM Error \\
\hline 11000 & 10 & 0.494071 & 0.212777 & -2.342335 & -0.974458 \\
18000 & 20 & 0.424634 & 0.165305 & -3.023494 & -1.177785 \\
27000 & 30 & 0.374365 & 0.15732 & -3.202855 & -1.355994 \\
64000 & 40 & 0.350142 & 0.138187 & -3.839098 & -1.454710 \\
125000 & 50 & 0.33173 & 0.135724 & -3.960688 & -1.536857 \\
216000 & 60 & 0.316112 & 0.128837 & -4.409357 & -1.612252 \\
343000 & 70 & 0.301227 & 0.13156 & -4.207334 & -1.689819 \\
512000 & 80 & 0.290315 & 0.130349 & -4.292186 & -1.750765 \\
729000 & 90 & 0.281466 & 0.128077 & -4.473879 & -1.803071 \\
1000000 & 100 & 0.273353 & 0.126315 & -4.641730 & -1.853556 \\
\hline
\end{tabular}

Table $2\left(X_{0}, Y_{0}\right)=(1.0,1.0), \operatorname{Tr} 1000=1.253903$

\begin{tabular}{llllll}
\hline M:No. of simmulation trials & n:No. of time steps & Symmetrization & PW EM & Sym Error & PW EM Error \\
\hline 18000 & 20 & 1.305908 & 1.22153 & -3.430431 & -2.956415 \\
27000 & 30 & 1.302775 & 1.235505 & -3.995513 & -3.018551 \\
64000 & 40 & 1.294995 & 1.234476 & -3.941091 & -3.191942 \\
125000 & 50 & 1.294358 & 1.2407 & -4.327311 & -3.207565 \\
216000 & 60 & 1.290095 & 1.23953 & -4.242404 & -3.318917 \\
343000 & 70 & 1.293495 & 1.247477 & -5.047403 & -3.229128 \\
512000 & 80 & 1.295325 & 1.249126 & -5.343942 & -3.183943 \\
729000 & 90 & 1.293081 & 1.248791 & -5.276165 & -3.239640 \\
1000000 & 100 & 1.290695 & 1.250402 & -5.654707 & -3.302475 \\
\hline
\end{tabular}




\section{Funding}

There's no funding

\section{Availability of data and materials}

The experiments are reproducible except the pseudo random numbers used in the Monte-Carlo simulation.

\section{Authors' contributions}

Introduced a hyperbolic version of Imamura-Ishigaki-Okumura's symmetrization, and by numerical experiments showed efficiency of the scheme. All authors read and approved the final manuscript.

\section{Competing interests}

The authors declare that they have no competing interests.

\section{Publisher's Note}

Springer Nature remains neutral with regard to jurisdictional claims in published maps and institutional affiliations.

Received: 12 October 2017 Revised: 10 November 2017 Accepted: 15 November 2017

Published online: 11 January 2018

\section{References}

1. Akahori, J, Barsotti, F, Imamura, Y: The Value of Timing Risk, working paper (2017). arXiv:1701.05695 [q-fin.PR]

2. Akahori, J, Imamura, Y: On symmetrization of diffusion processes. Quant. Finance. 14(7), 1211-1216 (2014)

3. Bowie, J, Carr, P: Static Simplicity. Risk. 7(8), 44-50 (1994)

4. Carr, P, Lee, R: Put-Call Symmetry: Extensions and Applications. Math. Finance. 19(4), 523-560 (2009)

5. Gobet, E: Weak approximation of killed diffusion using Euler schemes. Stoch. Process. Appl. 87(2), 167-197 (2000)

6. Hagan, P, Lesniewski, A, Woodward, D: Managing smile risk. Wilmott Mag. 1, 84-108 (2002)

7. Hagan, P, Lesniewski, A, Woodward, D: Probability Distribution in the SABR Model of Stochastic Volatility. In: Large Deviations and Asymptotic Methods in Finance Volume 110 of the series Springer Proceedings in Mathematics \& Statistics, pp. 1-35, (2015). http://www.springer.com/us/ book/9783319116044

8. Henry-Labordère, P: A General Asymptotic Implied Volatility for Stochastic Volatility Models (2005). arXiv:cond-mat/0504317

9. Hull, J: Options, Futures, and Other Derivatives. 9th edn. Pearson/Prentice Hall, Upper Saddle River (2014)

10. Imamura, Y, Ishigaki, Y, Okumura, T: A numerical scheme based on semi-static hedging strategy. Monte Carlo Methods Applic. 20(4), 223-235 (2014)

11. Imamura, Y, Takagi, $K$ : Semi-Static Hedging Based on a Generalized Reflection Principle on a Multi Dimensional Brownian Motion. Asia-Pacific Finan. Markets. 20(1), 71-81 (2013)

12. Kohatsu-Higa, A, Lejay, A, Yasuda, K: Weak approximation errors for stochastic differential equations with non-regular drift. J. Comput. Applied Math. 326, 138-158 (2017)

13. Ngo, H-L, Taguchi, D: Strong convergence for the Euler-Maruyama approximation of stochastic differential equations with discontinuous coefficients. Stat. Probab. Lett. 125, 55-63 (2017)

14. Taguchi, D: Stability Problem for One-Dimensional Stochastic Differential Equations with Discontinuous Drift. In: Donati-Martin, C, Lejay, A, Rouault, A (eds.) Séminaire de Probabilités XLVIII. Lecture Notes in Mathematics, vol 2168. Springer, Cham, (2016)

\section{Submit your manuscript to a SpringerOpen ${ }^{\circ}$ journal and benefit from:}

- Convenient online submission

- Rigorous peer review

- Open access: articles freely available online

- High visibility within the field

- Retaining the copyright to your article

Submit your next manuscript at $\gg$ springeropen.com 